\title{
Численное моделирование вольт-амперных характеристик двуслойной резистивной памяти на основе нестехиометрических оксидов металлов
}

\author{
(C) Г.М. Умнягин, В.Е. Дегтярев, С.В. Оболенский \\ Нижегородский государственный университет им. Н.И. Лобачевского, \\ 603950 Нижний Новгород, Россия \\ E-mail: Umnyagingm@gmail.com
}

Поступила в Редакцию 24 апреля 2019 г.

В окончательной редакции 29 апреля 2019 г.

Принята к публикации 29 апреля 2019 г.

\begin{abstract}
Проведено численное моделирование вольт-амперных характеристик структуры резистивной памяти на основе нестехиометрических оксидов тантала. В рамках исследования приводятся результаты проведения импульсного исследования структур с разной формой проводящего филамента, такой как усеченный конус с разным углом наклона образующей. Показано как форма и общий объем проводящего филамента сказываются на амплитуде тока и количестве импульсов, необходимом для полного процесса разрыва и восстановления филамента.
\end{abstract}

Ключевые слова: резистивная память, нестехиометрический, численное моделирование.

DOI: $10.21883 /$ FTP.2019.09.48138.21

\section{1. Введение}

Двухслойная резистивная память (RRAM) на основе оксидов тантала приобретает все большую популярность из-за перспективных преимуществ мемристров - приборов на ее основе в сравнении с традиционными видами памяти. Актуальность работы обусловлена необходимостью дальнейшего развития записывающих устройств и их характеристик, таких как плотность записи, быстродействие, энергонезависимость и пр. Известно, что образование и разрыв проводящих филаментов являются механизмом, который лежит в основе резистивного переключения, использующегося в RRAM для хранения и записи информации. Однако физическая модель, описывающая полную структуру RRAM, на настоящий момент не показана [1]. В работе используется полная численная модель электротермического резистивного переключения на основе моделирования дрейфа и диффузии вакансий кислорода в объеме структуры. Для численного решения используется пространственная дискретизация, основанная на методе конечных объемов. В используемой модели миграция вакансий кислорода определяется локальной температурой и электрическим полем, которые в свою очередь получены путем совместного решения уравнений непрерывности потока вакансий кислорода, теплопроводности и токостатики для двухслойной RRAM-структуры.

\section{2. Математическая модель}

Нами была исследована двухслойная резистивная память на основе нестехиометрического оксида тантала $\left(\mathrm{TaO}_{x}\right)$ и его пентооксида $\left(\mathrm{Ta}_{2} \mathrm{O}_{5}\right)$. Исследование проводилось при различных толщинах и формах проводящего филамента (рис. 1). Когда к изначальной структуре приложено большое отрицательное напряжение (процесс, называемый „формирование“), положительно заряженные вакансии кислорода притягиваются от нестехиометрического слоя к верхнему электроду, образуя проводящие филаменты.

Эти филаменты описываются как легированная область, где кислородные вакансии действуют в качестве легирующих примесей и, таким образом, определяют локальные значения электрической и тепловой проводимости.

Моделирование начинается сразу после процесса формирования. Поэтому в начальный момент времени структура имеет непрерывный проводящий филамент, который соединяет верхний электрод с нестехиометрическим слоем. После процесса формирования в зависимости от полярности приложенного к структуре напряжения проводятся процессы „сброса“ и „установки “ (reset/set), соответствующие записи в структуру состояний логического нуля или единицы соответственно. Оба процесса описываются посредством миграции кислородных вакансий, вызванной локальным электрическим полем и градиентом температуры из-за джоулевых нагревов. Таким образом, для моделирования данных процессов требуется самосогласованное решение трех уравнений в частных производных:

$$
\begin{gathered}
\frac{\partial n_{D}}{\partial t}=\nabla\left(D \nabla n_{D}-v n_{D}\right)+G, \\
\nabla \sigma \nabla \psi=0, \\
-\nabla k_{\mathrm{th}} \nabla T=J E=\sigma|\nabla \psi|^{2},
\end{gathered}
$$

где $G$ - слагаемое, описывающее генерацию процесса установки, $\sigma$ - электропроводность, $k_{\text {th }}-$ теплопро- 


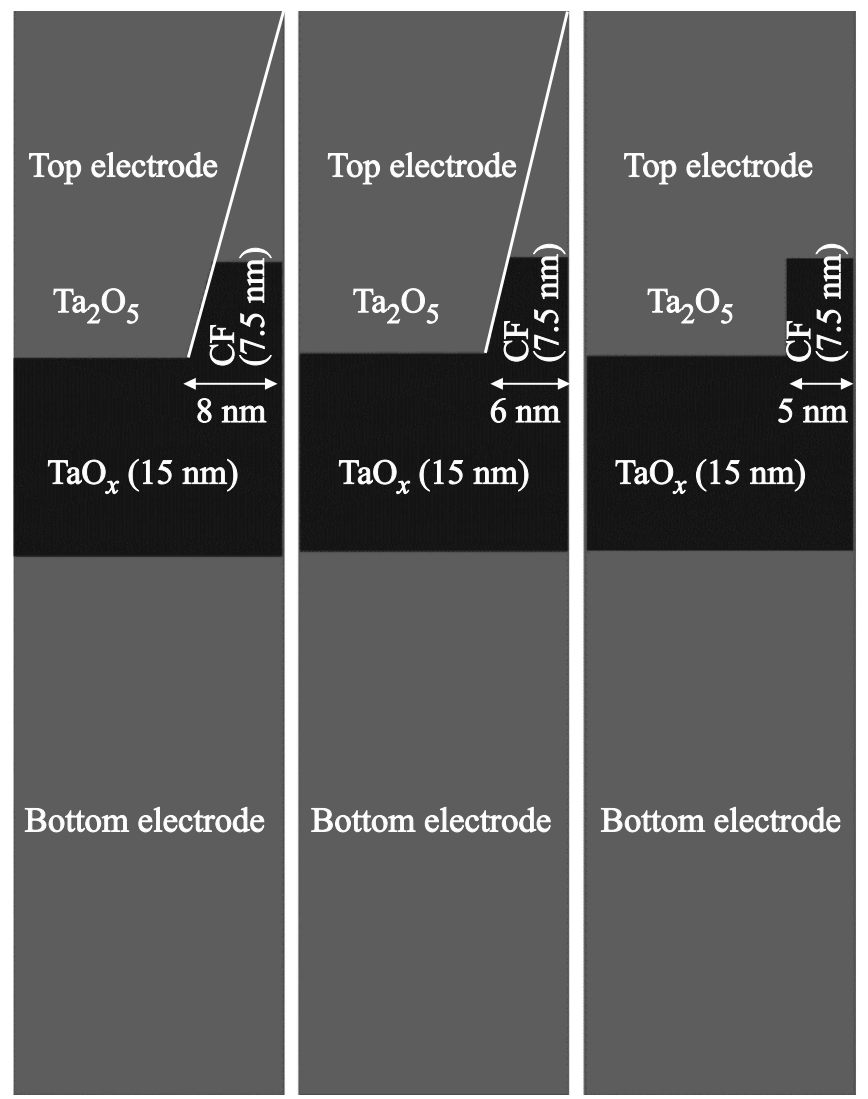

Рис. 1. Геометрические схемы рассматриваемых в работе структур в начальный момент времени.

водность. Кинетику процесса установки можно интерпретировать как мягкий пробой диэлектрика, связанный с миграцией или генерацией кислородных вакансий, вызванной преодолением работы выхода за счет температуры и приложенного напряжения. Предполагается, что скорость роста проводящего филамента пропорциональна скорости генерации ионов, которая дается выражением

$$
G=A \exp \left(-\frac{E_{b}-q V}{k T}\right)=A \exp \left(-\frac{E_{b}-q \beta E}{k T}\right),
$$

где $A$ - предэкспоненциальная константа, $E_{b}-$ энергетический барьер (работа выхода иона из решетки), $\beta-$ размер ячейки в моделировании. Слагаемое $q \beta E$ описывает снижение барьера, относящееся к прикладываемому полю. Это снижение заставляет кислородные вакансии двигаться вдоль направления электрического поля.

Была смоделирована область двухслойного RRAM на основе оксидов тантала. В расчетах осесимметричная модель позволила свести трехмерную задачу к двумерному решению в цилиндрических координатах. Материалы с активным оксидом контактируют с двумя электродами, также включенными в область численного решения (рис. 1). Граничными условиями для уравнения (2) являются $\psi=0$ и $\psi=V$ на нижнем и верхнем электро- дах соответственно. Внешние границы двух электродов определяются как идеальные теплоотводы с граничными условиями $T=300 \mathrm{~K}$, так как площадь электрода велика по отношению к проводящему филаменту.

Были проведены множественные численные эксперименты с различными параметрами моделирования (такими как теплоемкость, заряд кислородной вакансии, проводимость материалов, величина прикладываемого напряжения и т.д.), по итогам которых были построены характеристики зависимости тока через структуру от времени.

\section{3. Метод импульсного исследования}

Одним из основных уравнений, на которых базируется численное моделирование мемристров, - уравнение теплопроводности Фурье (3). В данной статье предложен метод частичного избавления от эффектов, связанных с нагревом структуры, таких как поперечная диффузия. В то время как математическая модель остается прежней, мы изменяем принцип измерения. Ранее была показана вольт-амперная характеристика с медленным и плавным изменением напряжения от времени [1].

В данной работе предлагается метод „импульсного исследования“. На структуру подается квадратный импульс длиной 0.1 мкс и величиной 3В. Далее 0.9 мкс идет „релаксация“ структуры, во время которой на $0.01 \mathrm{~B}$ идет установление температуры (рис. 2).

Для исследования было выбрано несколько характерных для данного эксперимента форм: прямой и перевернутый усеченные конусы и цилиндр, поскольку они правдоподобней описывают форму филамента после процесса формирования. В результате моделирования для филамента с формой прямого конуса, как и ожидалось, можно наблюдать сильное размытие боковой поверхности филамента из-за сильной диффузии в горизонтальном направлении. В силу необратимости про-

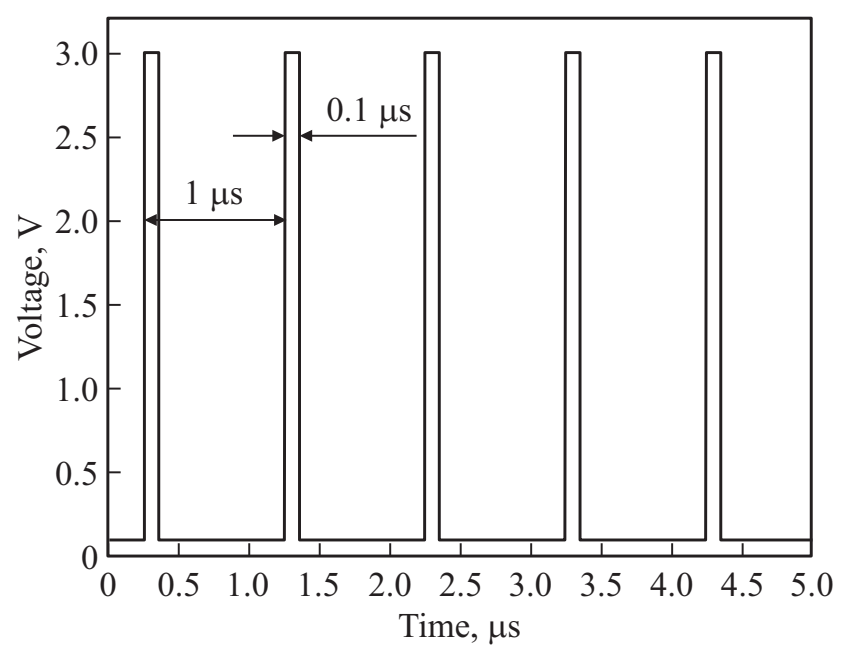

Рис. 2. Вид подаваемых на структуру импульсов. 


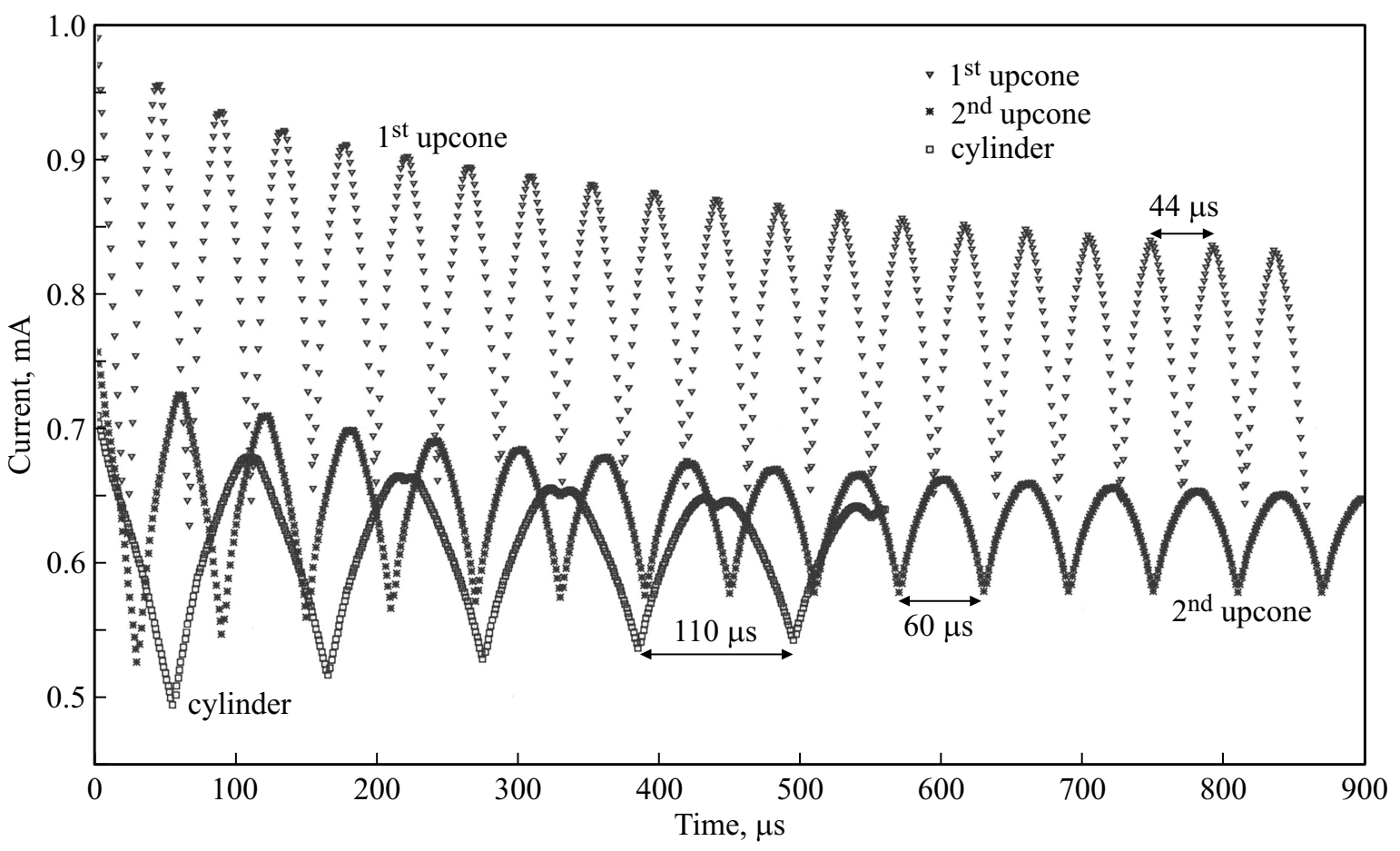

Рис. 3. Результаты импульсного исследования структур.

цесса диффузии филамент не будет полностью восстанавливаться. Поэтому хорошими результатами можно называть изменение тока на $20 \%$, так как уже при этом изменении можно однозначно определить, в каком состоянии находится филамент.

Для первой структуры (рис. 1) таких периодов импульсов было 22, для второй - 30. Было проверено, что такого количества достаточно для полного разрыва филамента в рассматриваемых структурах. Далее было выяснено, что именно такого же количества обратных импульсов (тех же, но с отрицательным значением напряжения) необходимо для процесса его восстановления.

На графиках, представленных на рис. 3, отмечены значения тока при напряжении 3В. На оси абсцисс отложено количество импульсов, численно равное времени моделирования в мкс (период следования импульсов 1 мкс). Видно, что минимальные и максимальные значения тока в процессах разрыва и восстановления филамента соответственно асимптотически выходят на определенный уровень.

\section{4. Обсуждение результатов}

Важность такого подхода к эксперименту можно отнести к вопросам технического толка. Это дополнительная возможность сделать процесс записи на устройство быстрее за счет, например, переменного напряжения на контактах. Иначе говоря, в то время как стационарное решение предлагает $10 \mathrm{c}$ на один цикл, импульсный метод сокращает это время до 44, 60 и 110 мкс для структур с различными формами (рис. 1).

Основываясь на результатах, приведенных на рис. 3, можно сделать вывод, что цилиндрическая форма филамента не самая удачная. Возможно, первые две структуры более хорошо описывают форму филамента, формирование которого происходит посредствам процесса „форминга“, описанного в работе [1]. Также можно предположить, что параметры выбраны не лучшим образом. Например, мы только предполагаем прямую зависимость теплопроводности от концентрации дефектов. Еще предстоит воспользоваться наработками статьи [2] для расширения предлагаемой базы материалов.

В любом случае стабилизация амплитуд колебания импульсов говорит о целесообразности предложенного метода. Существует альтернатива по определению состояния системы [3].

\section{5. Заключение}

Относительно удачные результаты исследования с помощью импульсного исследования могут лечь в основу принципиально новой ветви развития RRAM. Развитие может коснуться некоторых пунктов в технологии производства и использования резистивной памяти.

В дальнейшем мы планируем обратить свое внимание на ряд аспектов, которые не были учтены в исследовании статьи. Такие, как обеднение ионами области под филаментом после его формирования, нелинейная 
форма СF, подача синусоидального импульса вместо квадратного.

\section{Конфликт интересов}

Авторы заявляют, что у них нет конфликта интересов.

\section{Список литературы}

[1] S. Kim, S.-J. Kim, K.M. Kim, S.R. Lee, M. Chang, E. Cho, Y.B. Kim, Ch.J. Kim, U.-In Chung, I.-K. Yoo. Sci. Rept., 3, 1680 (2013).

[2] S. Kim, Sh. Choi, Wei Lu. ACS Nano, 8 (3), 2369 (2014).

[3] O. Cueto, A. Payet, Th. Cabout. Intern. Conf. Simulation of Semiconductor Processes and Devices (SISPAD), 45 (2014).

Редактор А.Н. Смирнов

\section{Numerical simulation of current-voltage characteristics of bi-layer resistive memory based on non-stoichiometric metal oxides}

G.M. Umnyagin, V.E. Degtyarov, S.V. Obolenskiy

Lobachevsky State University of Nizhny Novgorod, 603950 Nizhny Novgorod, Russia

Abstract A numerical simulation of the current-voltage characteristics of the structure of a resistive memory based on non-stoichiometric tantalum oxides has been carried out. The study presents the results of pulse response research of structures with different shapes of the conducting filament, such as a truncated cone with a different tilt angle. It is shown how the shape and total volume of the conducting filament affects the amplitude of the current and the number of pulses necessary for the complete process of breaking and restoring the filament. 